$\xi=-1$

\title{
Monophonic Wirelength in Graph Embedding
}

\author{
Sindhuja G. Michael ${ }^{1} *$ K. Uma Samundesvari ${ }^{2}$ \\ ${ }^{1}$ Department of Mathematics, Ponjesly College of Engineering, Nagercoil-629 003, Kanyakumari, Tamilnadu, India \\ ${ }^{2}$ Department of Mathematics, Noorul Islam Centre for Higher Education,Thuckalay-629 180, Kanyakumari, Tamilnadu, India \\ *Corresponding author E-mail:sindhujajefic@gmail.com
}

\begin{abstract}
In this paper, we define the monophonic embedding of graph $\mathrm{G}$ into another graph $\mathrm{H}$ and this paper presents a monophonic algorithm to find the monophonic wirelength of circulant networks $G[n, \pm S]$, where $S \subseteq\{1,2,3, \cdots, n / 2\}$ into the family of Cycle $C_{n}$, $n \geq 4$. The mono-phonic embedding of a graph $\mathrm{G}$ into a graph $\mathrm{H}$ is an embedding denoted by $\mathrm{f}_{\mathrm{mis}}$ a bijective map from the vertex set of $\mathrm{G}$ into the vertex set of $H$ and $f_{m}$ is a one-one mapping from the edge set $(x, y)$ of $G$ into $P_{m}(H)$ where $P_{m}(H)$ is the set of monophonic paths between $f_{m}(x)$ and $f_{m}(y)$ for every $f_{m}(x), f_{m}(y) \in H$. The monophonic wirelength of $f_{m}$ of $G$ into $H$ is the sum of distances of monophonic paths between two vertices $\mathrm{f}_{\mathrm{m}}(\mathrm{x})$ and $\mathrm{f}_{\mathrm{m}}(\mathrm{y})$ in $\mathrm{H}$ such that $(\mathrm{x}, \mathrm{y}) \in \mathrm{E}(\mathrm{G})$. In addition, the eccentricity, radius and diameter of an embedding of $\mathrm{G}$ into $\mathrm{H}$ are defined. The average wirelength of an embedding is defined and the bounds of average wirelength of some embeddings have been found.
\end{abstract}

Keywords:Circulant Networks; Congestion; Cycles; Embedding; Wirelength.

\section{Introduction}

For vertices $u$ and $v$ in a connected graph $G$, The distance $d(u, v)$ is the length of the shortest $u-v$ path in G. A chord of a path $u_{0}, u_{1}$, $u_{h}$ is an edge $u_{i} u_{j}$, with $j \geq i+[2]$. A u - v path is called a monophonic path if it is a chordless path. For two vertices $u$ and $v$ in a connected graph $G$, the monophonic distance $d_{m}(u, v)$ is the length of the longest $\mathrm{u}$ - v monophonic path in G. An u - v monophonic path of length $\mathrm{d}_{\mathrm{m}}(\mathrm{u}, \mathrm{v})$ is called an $\mathrm{u}-\mathrm{v}$ monophonic as stated in $[1,2]$.

By an embedding $\mathrm{f}: \mathrm{G} \rightarrow \mathrm{H}$ and a monophonic embedding $\mathrm{f}_{\mathrm{m}}$ : $\mathrm{GH}$, it is meant that the graphs $\mathrm{G}(\mathrm{V}, \mathrm{E})$ and $\mathrm{H}(\mathrm{V}, \mathrm{E})$ are finite, simple and connectedwith $\mathrm{n}$ vertices. Given a host graph $\mathrm{H}$, which represents the network into which other networks are to be embedded, and a guest graph $\mathrm{G}$, which represents the network to be embedded, the problem is to find a mapping from $V(G)$ to $V(H)$ such that each edge of $\mathrm{G}$ can be mapped to a path in Has given in [3-7]. An embedding $f$ of $G$ into $\mathrm{H}$ is defined as follows:

1) $f$ is a bijective map from $V(G)$ to $V(H)$.

2) $f$ is an one-to-one mapping from $E(G)$ to $P_{f}(f(u), f(v))$ where $P_{f}(f(u), f(v))$ is a path in $H$ between $f(u)$ and $f(v)$ for $(u, v) \in$ $\mathrm{E}(\mathrm{G})$ as defined in [8-10].

An embedding $f_{m}: G \rightarrow H$ is called a monophonic embedding if $f_{m}$ maps each vertex of $\mathrm{G}$ into a vertex of $\mathrm{H}$ and each edge (x, y) of $\mathrm{G}$ is mapped to a monophonic path between $f_{m}(x)$ and $f_{m}(y)$ in $H$. The edge congestion of an embedding $\mathrm{f}$ of $\mathrm{G}$ into $\mathrm{H}$ is the maximum number of edges of the graph $G$ that are embedded on any single edge of $\mathrm{H}$. The wirelength of an embedding $f$ of $\mathrm{G}$ into $\mathrm{H}$ is given by,

$$
\begin{array}{r}
W L_{f}(G, H)=\sum_{(u, v) \in E(G)} d_{H}(f(u), f(v)) \\
=\sum_{e \in E(H)} E C_{f}(G, H(e))
\end{array}
$$

The wirelength problem of a graph $\mathrm{G}$ into $\mathrm{H}$ is to find an embedding of $\mathrm{G}$ into $\mathrm{H}$ that induces the minimum wirelength $\mathrm{WL}(\mathrm{G}, \mathrm{H})$ as defined in [6],[11], [12].

\section{Preliminaries}

In this section, we have given definition, example, Lemma and Theorem which are needed in the sequel.

Definition 2.1: $f_{m}: G \rightarrow H$ be an monophonic embedding. The monophonic wirelength $M W L(G, H)$ of $f_{m}$ is given by, $\mathrm{MWL}_{\mathrm{f}_{\mathrm{m}}}(\mathrm{G}, \mathrm{H})=$ $\sum_{(\mathrm{x}, \mathrm{y}) \in \mathrm{E}(\mathrm{G})} \mathrm{d}_{\mathrm{m}}\left(\mathrm{f}_{\mathrm{m}}(\mathrm{x}), \mathrm{f}_{\mathrm{m}}(\mathrm{y})\right)$

Definition 2.2: Let $f_{m}: G \rightarrow H$ be a monophonic embedding. The monophonic edge congestion off $m$ of $G$ into $H$ is the maximum number of edges of the graph $G$ that are embedded on an edge $e \in H$ and is given by, $M_{E} C_{f_{m}}(G, H)=\max M E C_{f_{m}}(G, H(e))$. The monophonic wirelength problem of a graph $G$ into $H$ is the problem of finding a monophonic embedding $f_{m}: G \rightarrow H$ that produces the monophonic wirelength $M W L(G, H)$.

Definition 2.3: The eccentricity of a vertex of graph $G$ into $H$ of an embedding $f$ is given by ef $(v)=\max \{d(f(u), f(v)) /(u, v) \in E(G)$ and $P_{f}(f(u), f(v))$ is a path in $H$ for every $(u, v) \in E(G)$ and for any vertex u in $G$ \}.

Definition 2.4: The radius of $f: G \rightarrow H$ is given by $r_{f}(G, H)=$ $\min \left\{e_{f}(v) / v \in V(G)\right\}$ and the diameter of $f: G \rightarrow H$ is given by $d_{f}(G, H)=\max \left\{e_{f}(v) / v \in V(G)\right\}$.

Definition 2.5: The monophonic eccentricity of a vertex of graph $G$ into $H$ of an embedding $f_{m}$ is given by $\mathrm{e}_{\mathrm{f}_{\mathrm{m}}}(\mathrm{v})=$ 
$\max \left\{\mathrm{d}_{\mathrm{m}}\left(\mathrm{f}_{\mathrm{m}}(\mathrm{u}), \mathrm{f}_{\mathrm{m}}(\mathrm{v})\right) /(\mathrm{u}, \mathrm{v}) \in \mathrm{E}(\mathrm{G})\right.$ and $\mathrm{P}_{\mathrm{f}_{\mathrm{m}}}\left(\mathrm{f}_{\mathrm{m}}(\mathrm{u}), \mathrm{f}_{\mathrm{m}}(\mathrm{v})\right)$ is a path in $H$ for every $(u, v) \in E(G)$ and for any vertex $u$ in $G\}$.

Definition 2.6: The monophonic radius of $f_{m}: G \rightarrow H$ is given $\operatorname{byr}_{f_{m}}(G, H)=\min \left\{e_{f_{m}}(v) / v \in V(G)\right\}$ by and the diameter of $f_{m}$ : $G \rightarrow H$ is given by $d m_{f_{m}}(G, H)=\max \left\{e_{f_{m}}(v) / v \in V(G)\right\}$.

Lemma 2.7: (Congestion lemma): Let $G$ be an r-regular graph and let $f: G \rightarrow H$ be an embedding. Let the graph $H \backslash E$ has the components $H_{i}, i=1,2$ and $G_{i}=f^{1}\left(H_{i}\right)$ then the edge cut $E$ of $H$ has the following properties:

1) The path $\mathrm{P}_{\mathrm{f}}(\mathrm{f}(\mathrm{x}), \mathrm{f}(\mathrm{y}))$ has no edges in $\mathrm{E}$ for every edge (x, y) $\in \mathrm{G}_{\mathrm{i}}, \mathrm{i}=1,2$.

2) The path $\operatorname{Pf}(f(x), f(y))$ has exactly one edge in $E$ for every edge $(x, y)$ in $G$ with $x \in G_{1}$ and $y \in G_{2}$.

3) $G_{1}$ is a maximum subgraph of $G$.

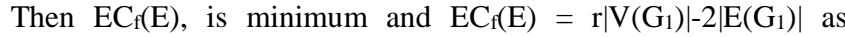
proved in [6].

Lemma 2.8: (Partition Lemma): Let $f: G \rightarrow H$ be an embedding. Let $\left\{E_{1}, E_{2}, \ldots, E_{p}\right\}$ be a partition of $E(H)$ such that each $E_{i}$ is an edge cut of $H$. Then $W L_{f}(G, H)=\sum_{i=i}^{p} E C_{f}\left(E_{i}\right)$ as proved in [6].

Lemma 2.9: ( $k$-partition Lemma): Let $f$ be an embedding of $G$ into $H$. Let $\left\{E_{1}, E_{2}, \ldots, E_{p}\right\}$ be a partition of $k[E(H)]$ such that each $E_{i}$ is an edge cut of $H$. Then $\mathrm{WL}_{\mathrm{f}}(\mathrm{G}, \mathrm{H})=\frac{1}{\mathrm{k}} \sum_{\mathrm{i}=\mathrm{i}}^{\mathrm{p}} \mathrm{EC}_{\mathrm{f}}\left(\mathrm{E}_{\mathrm{i}}\right)$ as proved in [13].

Lemma 2.10: (Generalized partition Lemma): Let f be an embedding of $G$ into $H$. For $1 \leq i \leq k$, suppose $S_{i}=\left\{S_{1}{ }_{1}, S_{2}{ }_{2}, \ldots, S_{p i}{ }\right\}$ partitions $E(H) \backslash F_{i}$ for mutually disjoint $F_{i}$ 's such that $S_{j,}^{i}, 1 \leq j \leq p_{i}, 1 \leq i$ $\leq k$ and $S=\bigcup_{i=1}^{k} F_{i}$ are all edge cuts of $H$. Then $W L_{f}(G, H)=$ $\frac{1}{k}\left[\sum_{i=1}^{k} \sum_{j=1}^{p_{i}} E C_{f}\left(S_{j}^{i}\right)+E C_{f}(S)\right]$ as proved in [5].

Lemma 2.11: (Monophonic congestion Lemma): Let $G$ be an $r$ regular graph with $n$ vertices. Let $H$ be a finite graph with $n$ vertices. Let $f_{m}: G \rightarrow H$ be a monophonic embedding of $G$ into $H$. Let the graph $H \backslash E_{j}, j=1,2, \ldots, p ; 0<p<|E(G)|$, have the components $H_{i}, i=1,2$ and $G_{i}=f_{m}^{-1}\left(H_{i}\right)$, where $E_{j}$ 's are the edge cuts of $H$, form a partition in $H$ and have the following properties:

1) For $m \geq 0$, there are $m$ edges $(x, y) \in G_{i}, i=1,2$, such that the monophonic path $\mathrm{P}_{\mathrm{f}_{\mathrm{m}}}\left(\mathrm{f}_{\mathrm{m}}(\mathrm{x}), \mathrm{f}_{\mathrm{m}}(\mathrm{y})\right)$ has exactly two edges in $\mathrm{E}_{\mathrm{j}}$.

2) The monophonic path $P_{f_{m}}\left(f_{m}(x), f_{m}(y)\right)$ has exactly one edge in $E_{j}$ for every $(x, y) \in G$ with $x \in G_{1} \& y \in G_{2}$.

Where

$\mathrm{G}_{1}$ is a maximum subgraph of $\mathrm{G}$. Then $M E C_{f_{m}}\left(E_{j}\right)$ is monophonic and the monophonic wirelength of $\mathrm{f}_{\mathrm{m}}$ of $\mathrm{G}$ into $\mathrm{H}$ is given byMWL $_{\mathrm{f}_{\mathrm{m}}}(\mathrm{G}, \mathrm{H})=\sum_{\mathrm{j}=1}^{\mathrm{p}} \mathrm{MEC}_{\mathrm{f}_{\mathrm{m}}}\left(\mathrm{E}_{\mathrm{j}}\right)$, where the monophonic edge congestion, $\operatorname{MEC}\left\{f_{m}\right\}\left(E_{j}\right)=r\left|V\left(G_{l}\right)\right|-2\left|E\left(G_{l}\right)\right|+2 m, m \geq 0$.

Theorem 2.12:Let $f: G \rightarrow H$ be an embedding, where $G$ be the circulant graph $G[2 n, \pm S], S \subseteq\{1,2,3, \ldots, n\}$ and $H$ be the grid $M[n \times 2]$. If $d$ is the diameter of the embedding of $G$ into $H$, then,

1) $\quad \mathrm{WL}(\mathrm{G}[2 \mathrm{n},\{1,2, \ldots, \mathrm{n}-1\}], \mathrm{M}[\mathrm{n} \times 2])=2 \mathrm{n}|\mathrm{S}|+1 / 3[\mathrm{~d}(\mathrm{~d}-1)$ (2d-1)].

2) $\mathrm{WL}(\mathrm{G}[2 \mathrm{n},\{1,2, \ldots, \mathrm{n}\}], \mathrm{M}[\mathrm{n} \times 2])=(2 \mathrm{n}|\mathrm{S}| 1)-\mathrm{n}+1 / 3[\mathrm{~d}(\mathrm{~d}-$ 1)(2d-1)].

3) $\mathrm{WL}(\mathrm{G}[2 \mathrm{n},\{1,2, . ., \mathrm{n}-2\}], \mathrm{M}[\mathrm{n} \times 2])=2(|\mathrm{~S}|-1)(\mathrm{n}-1)+1 / 3[\mathrm{~d}(\mathrm{~d}-$ 1)(2d-1)].

\section{Average wirelength}

The average wirelength of an embedding $\mathrm{f}: \mathrm{G} \rightarrow \mathrm{H}$ denoted by $\mu \mathrm{W}_{\mathrm{f}}(\mathrm{G}, \mathrm{H})$,is the expected edge congestion of $\mathrm{G}$ into $\mathrm{H}$ between a randomly chosen pair of distinct vertices $f(x), f(y) \in H$ such that $(\mathrm{x}, \mathrm{y}) \in \mathrm{E}(\mathrm{G})$. Therefore $\mu W_{f}(G, H)=$

$\frac{1}{|E(G)|} \sum_{(x, y) \in E(G)} d_{H}(f(x), f(y))=\frac{1}{|E(G)|} \sum_{e \in E(H)} E C_{f}(G, H(e))$

In the same manner, the average monophonic wirelength of

$\mathrm{f}_{\mathrm{m}}: \mathrm{G} \rightarrow \mathrm{H}$ is given by,

$\mu M W_{f_{m}}(G, H)=\frac{1}{|E(G)|} \sum_{e \in E(H)} M E C_{f_{m}}(G, H(e))$.

\subsection{Bounds on average wirelength and average mono- phonic wire length}

For $n \geq 2$

$2 \leq \mu W_{f}(G[2 n,\{1,2,3, \ldots, n-1\}], M[n \times 2]) \leq[n / 2]$ and

$2 \leq \mu M W_{f_{m}}(G[2 n,\{1,2,3, \ldots, n-1\}], M[n \times 2]\} \leq\left[\frac{n+1}{2}\right]$

For $n \geq 3$,

$2 \leq \mu W_{f}(G[2 n,\{1,2,3, \ldots, n-2\}], M[n \times 2]) \leq[n / 4]$ and

$2 \leq \mu M W_{f_{m}}(G[2 n,\{1,2,3, \ldots, n-2\}], M[n \times 2]\} \leq\left[\frac{n}{3}\right]$.

For $n \geq 4$,

$2 \leq \mu W_{f}(G[2 n,\{1,2,3, \ldots, n-3\}], M[n \times 2]) \leq\left[\frac{n-1}{4}\right]$ and

$2 \leq \mu \operatorname{MW}_{\mathrm{f}_{\mathrm{m}}}(\mathrm{G}[2 \mathrm{n},\{1,2,3, \ldots, \mathrm{n}-3\}], M[\mathrm{n} \times 2]\} \leq\left[\frac{\mathrm{n}-1}{3}\right]$

\section{Monophonic wirelength of circulant net- works into cycles}

Definition 4.1:A connected undirected graph represented by $G$ [n, $\pm S]$ where $S \subseteq\{1,2,3, \ldots,[n / 2]\}, n \geq 3$ is said to be a circulant graph if it consists of the vertex set.

$V=\{0,1,2 \ldots, n-1\}$ and the edge set

$E=\{(x, y):|x-y| \cong s(\bmod n), s \in S\}$ as defined in [5].

Definition 4.2: $A$ graph with a closed walk consisting of $n$ points is called a cycle, denoted by $C_{n}$ as defined in [14].

Theorem 4.3: $A$ set of $k$ consecutive vertices of $G[n, \pm 1]$; $1 \leq k \leq n$ induces a maximum subgraph of $G[n, \pm S]$ Where $S \subseteq\{1,2,3, \ldots, j\}, 1 \leq j \leq[n / 2], n \geq 3$ as defined in [5].

In this section, $G$ denotes the circulant graph $G[n, \pm S]$ where $S$ $\subseteq\{1,2,3, \ldots,[n / 2]\}$ of $n$ vertices and $H$ denotes the cycle graph $C_{n}$ with $n$ vertices and $f_{m}$, the monophonic embedding from $\mathrm{G}$ to $\mathrm{H}$.

Theorem 4.4: If $f_{m}: G \rightarrow H$ is a monophonic embedding, then the wirelength induced by $f_{m}$ from the Circulant graph to Cycle graph is monophonic.

Proof:

We prove this for two cases.

Case (i) ( $\mathrm{n}$ is odd)

Let $P_{1}=\left\{P_{1}^{1}, P_{2}^{1}, \ldots, P_{\underline{n-1}}^{1}\right\}$ and $P_{2}=\left\{P_{1}^{2}, P_{2}^{2}, \ldots, P_{\underline{n-1}}^{2}\right\}$ where $P_{S}^{1}=$ $\left\{(s-1, s),\left(\frac{(n-3)}{2}+s, \frac{(n-1)}{2}+s\right)\right\}$ and $P_{s}^{2}=\left\{(s-1, s),\left(\frac{(n-1)}{2}+\right.\right.$ $\left.\left.s, \frac{(n+1)}{2}+s\right)\right\}, 1 \leq \mathrm{s} \leq \frac{(n-1)}{2}$, taken modulo $\mathrm{n}$.

Consider the edges, $\mathrm{R}_{1}=\{(\mathrm{n}-1,0)\}$ and $R_{2}=\left\{\left(\frac{(n-1)}{2}, \frac{n+1}{2}\right)\right\}$, clearly $P_{t}$ partitions $E(H) \backslash R_{t}, t=1,2$ and the sets $R_{1}$ and $R_{2}$ are disjoint and their union is an edge cut of $\mathrm{H}$. For each $\mathrm{s}, \mathrm{E}(\mathrm{H}) \backslash P_{s}^{t}$ has two components $H_{S_{1}}^{t}$ and $H_{S_{2}}^{t}$ induced by consecutive vertices on $\mathrm{H}$ with $\left|H_{s_{1}}^{t}\right|=\left[\frac{n}{2}\right]=\left|H_{s_{2}}^{t}\right|$. Let $G_{s_{1}}^{t}=f_{m}^{-1}\left(H_{s_{1}}^{t}\right)$ and $\mathrm{G}_{\mathrm{s}_{2}}^{\mathrm{t}}=$ $\mathrm{f}_{\mathrm{m}}^{-1}\left(\mathrm{H}_{\mathrm{s}_{2}}^{\mathrm{t}}\right)$.Then $G_{s_{1}}^{t}$ is on $\left[\frac{n}{2}\right]$ consecutive vertices of $\mathrm{G}[\mathrm{n}, \pm 1]$ and these vertices induce a maximal subgraph of $\mathrm{G}[\mathrm{n}, \pm \mathrm{S}]$ by theorem 4.3. Hence $P_{S}^{t}$ satisfies the monophonic congestion lemma and therefore $M E C_{f_{m}}\left(P_{s}^{t}\right)$ is monophonic and thus the wire length induced by $\mathrm{f}_{\mathrm{m}}$ is monophonic.

Case (ii) ( $\mathrm{n}$ is even) 
Let $\mathrm{P}_{\mathrm{t}}, \mathrm{t}=1,2, \ldots, \mathrm{n} / 2$ be the edge cuts of $\mathrm{H}$, form a partition in $\mathrm{H}$. For each $\mathrm{t}$, let $P_{t}=\left\{(t-1, t),\left(\frac{n}{2}+t-1\right),\left(\frac{n}{2}+t\right)\right\} 1 \leq \mathrm{t} \leq \mathrm{n} / 2$ where the vertices are taken modulo $n$. That is $P_{t}$ has two direct opposite edges of the cycle graph $\mathrm{H}$. Let $H_{t_{1}}$ and $H_{t_{2}}$ are the components of $\mathrm{H} \backslash \mathrm{P}_{\mathrm{t}}$ for each $\mathrm{t}$. Let $\mathrm{G}_{\mathrm{t}_{1}}=\mathrm{f}_{\mathrm{m}}^{-1}\left(\mathrm{H}_{\mathrm{t}_{1}}\right)$ and $\mathrm{G}_{\mathrm{t}_{2}}=\mathrm{f}_{\mathrm{m}}^{-1}\left(\mathrm{H}_{\mathrm{t}_{2}}\right)$. Then the graphs $G_{t_{1}}$ and $G_{t_{2}}$ are on $\left[\frac{n}{2}\right]$ consecutive points of $\mathrm{G}[\mathrm{n}$, $\pm 1]$ and these points induce a maximal subgraph of $G[n, \pm S]$ by theorem 4.3. Hence, $\mathrm{P}_{\mathrm{t}}$ satisfies the monophonic congestion lemma and therefore $M E C_{f_{m}}\left(P_{t}\right)$ is monophonic and thus the wirelength induced by $\mathrm{f}_{\mathrm{m}}$ is monophonic.

\section{Monophonic Embedding Algorithm}

Aim: To find a monophonic embedding $\mathrm{f}_{\mathrm{m}}: \mathrm{G} \rightarrow \mathrm{H}$ that produces the monophonic wirelength $M E C_{f_{m}}(G, H)$ where $\mathrm{G}$ is the family of circulant graph with $\mathrm{n}$ vertices of $\mathrm{r}$-regular and $\mathrm{H}$ is the cycle graph $C_{n}, n \geq 4$.

\subsection{Monophonic Algorithm}

1) Name the vertices of $G[n, \pm S], S \subseteq\{1,2,3, \ldots, n / 2\}$ as the vertices of Cycle from $0,1,2, \ldots, \mathrm{n}-1$.

2) Name the vertices of $C_{n}$ as $0,1,2, \ldots, n-1$ in clockwise. Input: A family of circulant graphs $\mathrm{G}[\mathrm{n}, \pm \mathrm{S}], \mathrm{S} \subseteq\{1,2,3, \ldots, \mathrm{n} / 2\}$ and the cycle graph $\mathrm{C}_{\mathrm{n}}$.

Output: A monophonic embedding $f_{m}$ from $G[n, \pm S]$, into $C_{n}$ given by $\mathrm{f}_{\mathrm{m}}(\mathrm{x})=\mathrm{x}$ with monophonic wirelength $\operatorname{MWL}\left(\mathrm{G}[\mathrm{n}, \pm \mathrm{S}], \mathrm{C}_{\mathrm{n}}\right)$ $=\left[\frac{n}{2}\right]\left[M E C_{f_{m}}(P)\right]$.

Proof: In theorem 4.4, it is proved that the wirelength induced by $f_{m}$ from $G[n, \pm S]$ to $C_{n}$ is monophonic. We prove two cases.

Case (i) ( $n$ is odd)

Using the notations used in case (i) of theorem 4.4, we have by generalized partition lemma,

$$
M W L\left(G[n, \pm S], C_{n}\right)=\frac{1}{2}\left\{\sum_{t=1}^{2} \sum_{s=1}^{\frac{(n-1)}{2}} M E C_{f_{m}}\left(P_{s}^{t}\right)+M E C_{f_{m}}(P)\right\}
$$

As $\mathrm{H} \backslash \mathrm{P}$ is isomorphic to $\mathrm{H} \backslash P_{S}^{t}$,

$$
\begin{aligned}
M W L\left(G[n, \pm S], C_{n}\right) & =\frac{1}{2}\left\{(n-1) M E C_{f_{m}}(P)+M E C_{f_{m}}(P)\right\} \\
& =\frac{n}{2} M E C_{f_{m}}(P)
\end{aligned}
$$

Case (ii) ( $\mathrm{n}$ is even)

Using the notations used in case (ii) of theorem 4.4, we have by generalized partition lemma,

$$
\begin{aligned}
\operatorname{MWL}\left(G[n, \pm S], C_{n}\right) & =\frac{1}{2}\left\{\sum_{t=1}^{\frac{n}{2}} M E C_{f_{m}}\left(P_{t}\right)\right\} \\
& =\frac{1}{2}\left\{n M E C_{f_{m}}(P)\right\}=\frac{n}{2} M E C_{f_{m}}(P)
\end{aligned}
$$

\subsubsection{Monophonic algorithm (i)}

Input: A family of circulant graph $G\left[n,\left\{1,2, \ldots,\left[\frac{n}{2}-1\right]\right\}\right]$ and the cycle graph $\mathrm{C}_{\mathrm{n}}, \mathrm{n} \geq 4$.

Output: A monophonic embedding fmfrom $G\left[n,\left\{1,2, \ldots,\left[\frac{n}{2}-\right.\right.\right.$ 1]\}] into $\mathrm{C}_{\mathrm{n} g i v e n}$ by $\mathrm{f}_{\mathrm{m}}(\mathrm{x})=\mathrm{x}$ with monophonic wirelength,

$$
\begin{aligned}
\operatorname{MWL}(G[n,\{1,2, \ldots, & {\left.\left.\left[\frac{n}{2}-1\right]\right\}, C_{n}\right) } \\
& =\left\{\begin{array}{l}
\frac{n}{2}\left(3|s|^{2}-|s|\right) \text { ifniseven } \\
\frac{n}{2}\left(3|s|^{2}-3|s|+2\right) \text { ifnisodd }
\end{array}\right.
\end{aligned}
$$

\subsubsection{Monophonic algorithm (ii)}

Input: A family of circulant graphG $\left[\mathrm{n},\left\{1,2, \ldots,\left[\frac{\mathrm{n}}{2}-2\right]\right\}\right]$, and the cycle graph $\mathrm{C}_{\mathrm{n}}, \mathrm{n} \geq 5$.

Output: A monophonic embedding $\mathrm{f}_{\mathrm{m}}$ from $\mathrm{G}\left[\mathrm{n},\left\{1,2, \ldots,\left[\frac{\mathrm{n}}{2}-2\right]\right\}\right]$ into $\mathrm{C}_{\mathrm{n}}$ given by $\mathrm{f}_{\mathrm{m}}(\mathrm{x})=\mathrm{x}$ with monophonic wirelength,

$$
\begin{aligned}
\operatorname{MWL}\left(G\left[n,\left\{1,2, \ldots,\left[\frac{n}{2}-2\right]\right\}, C_{n}\right)\right. \\
=\left\{\begin{array}{l}
\frac{n}{2}\left(3|s|^{2}-3|s|-4\right. \text { ifniseven } \\
\frac{n}{2}\left(3|s|^{2}-|s|-2\right. \text { ifnisodd }
\end{array}\right.
\end{aligned}
$$

\subsubsection{Monophonic algorithm (iii)}

Input: A family of circulant graph $G\left[n,\left\{1,2, \ldots,\left[\frac{n}{2}-3\right]\right\}\right]$, and the cycle graph $\mathrm{C}_{\mathrm{n}}, \mathrm{n}>6$.

Output: A monophonic embedding $\mathrm{f}_{\mathrm{m}}$ from

$\mathrm{G}\left[\mathrm{n},\left\{1,2, \ldots,\left[\frac{\mathrm{n}}{2}-3\right]\right\}\right]$ into $\mathrm{C}_{\mathrm{n}}$ given by $\mathrm{f}_{\mathrm{m}}(\mathrm{x})=\mathrm{x}$ with monophonic wirelength,

$$
\begin{aligned}
M W L\left(G\left[n,\left\{1,2, \ldots .,\left[\frac{n}{2}-3\right]\right\}, C_{n}\right)\right. \\
=\left\{\begin{array}{l}
\frac{n}{2}\left(3|s|^{2}+7|s|-8\right) \text { ifniseven } \\
\frac{n}{2}\left(3|s|^{2}+5|s|-6\right) \text { ifnisodd }
\end{array}\right.
\end{aligned}
$$

\section{Conclusion}

In this paper we have applied the monophonic idea on graph embedding of two graphs and also we have given a monophonic algorithm for finding the monophonic wirelength of circulant networks into cycles.

\section{Acknowledgement}

The authors thank the anonymous referee for the valuable suggestions in revising the manuscript.

\section{References}

[1] F. Buckley and F. Harary, Distance in Graph, Addison- Wesley, California, 1990.

[2] A.P. Santhakumaran and P. Titus, "Monophonic Distance in Graphs", Discrete Mathematics Algorithms Application, Vol.3, No.2, (2011), pp.159-169, https://doi.org/10.1142/S1793830911001176.

[3] P. Maya and T. Nicholas, "Some Results on Integer Edge Cordial Graph", DJ Journal of Engineering and Applied Mathematics, Vol.3, No.1, (2017), pp. 1-12, https://doi.org/10.18831/djmaths.org/2017011001.

[4] P. Manuel, "Minimum Average Congestion of Enhanced and Augmented Byper-Cube into Complete Binary Tree", Discrete Applied Mathematics, Vol.159, No.5, (2010), pp.360-366, https://doi.org/10.1016/j.dam.2010.12.001.

[5] IndraRajasingh, Paul Manuel, M. Arockiaraj and BharatiRajan, "Embeddings of Circulant Networks", Journal of Combinatorial Optimization, Vol.26, No.1, (2013), pp.135-151, https://doi.org/10.1007/s10878-011-9443-x.

[6] P. Manuel, I. Rajasingh, B. Rajan and H. Mercy, "Exact Wirelength of Hypercube on a Grid", Discrete Applied Mathematics, Vol.157, 
No.7,

(2009),

pp.1486-

1495.https://doi.org/10.1016/j.dam.2008.09.013.

[7] Jess Mathew, "Subdivided Stars Super (b, d) - Edge-Antimagic Total Graph Labelling", DJ Journal of Engineering and Applied Mathematics, Vol.1, No.1, (2015), pp.30-34, https://doi.org/10.18831/djmaths.org/2015011005.

[8] S.L. Bezrukov, J.D. Chavez, L. H. Harper, M. Rottger and U. P.Schroeder, "Embedding of Hypercubes into Grids", International Symposium on Mathematical Foundations of Computer Science, Vol.1450, (1998), pp.693-701, https://doi.org/10.1007/BFb0055820.

[9] L. Bezrukov, "Embedding Complete Trees into the Hypercube", Discrete Applied Mathematics, Vol.110, No.2-3, (2001), pp.101119,https://doi.org/10.1016/S0166-218X(00)00256-0.

[10] JasinthaQuadras, S. Sarah Surya, "Linear Wirelength of Circulant Networks", International Journal of Pure and Applied Mathematics, Vol.86, No.6, (2013), pp.883-891,

[11] P. Manuel, M. Arockiaraj, I. Rajasingh and B. Rajan, "Embedding Hypercubes into Cylinders, Snakes and Caterpillars for Minimizing Wirelength", Discrete Applied Mathmetics, Vol.159, No.17, (2011), pp.2109-2116, https://doi.org/10.1016/j.dam.2011.07.003

[12] I. Rajasingh, J. Quadras, P.Manuel and A.William, "Embedding of Cycles and Wheels into Arbitrary Trees", Networks, Vol.44, (2004) pp.173-178, https://doi.org/10.1002/net.20027.

[13] Arokiaraj, Paul Manuel, IndraRajasingh and BharatiRajan, "Wirelength of 1-Fault Hamiltonian Graphs into Wheels and Fans", In formation Processing Letters, Vol.111, No.18, (2011), pp.921925, https://doi.org/10.1016/j.ipl.2011.06.011.

[14] F. Harary, Graph Theory, Addison- Wesley, 1969.https://doi.org/10.21236/AD0705364. 